\title{
EXTRUSIÓN DE SORGO INTEGRAL Y DECORTICADO*
}

\author{
B.F. Martínez**, C. L.Pau***
}

\section{RESUMEN}

El presente trabajo tuvo como objetivos fundamentales la elaboración de productos instantáneos utilizando como materia prima sorgo blanco (ISIAP-Dorado) integral y decorticado, con tres diferentes contenidos de humedad (12, 15 y 18\%). Los productos expandidos obtenidos no fueron afectados en sus contenidos de proteína, grasa, fibra y cenizas. El color, de los productos expandidos de sorgo decorticado, mostró mayores valores en relación a los obtenidos con sorgo integral y las propiedades funcionales de viscosidad e índices de absorción y solubilidad en agua fueron modificadas capacitando estos productos a nuevos y variados usos. La densidad y grado de expansión aumentó a medida que aumentó el contenido de humedad de las harinas, con una mayor expansión y menor densidad de los productos obtenidos de sorgo decorticado. La prueba sensorial de aceptación (no mostró diferencia significativa entre tratamientos en los parámetros de sabor, textura y apariencia general.

\begin{abstract}
The main objective of this study was to manufacture instant products using whole and decerticated white sorghum (ISIAP-Dorado) with three different humidity contents $(12,15$ and $18 \%)$. The protein, fat, fiber and ash contents were not affected by the process. The color of the extuded products from the decorticated sorghum showed higher values with respect to those of the whole sorghum. The viscosity properties, absorption indexes and water solubility of these products were modified, rendering them to new and varied uses. The density and degree of extrusion increased along with the increased humidity content of the flours, obtaining a larger extrusion and lower density for the products of decorticated sorghum. The palatability trial conducted with 50 persons showed no significant differences among treatments for the parameters of flavor, texture and overall appearance.
\end{abstract}

\section{INTRODUCCIÓN}

En América Latina el cultivo del sorgo se ha constituído en una especie de gran importancia atribuído, principalmente, al hecho que es un cereal que puede tolerar, tanto climas áridos como húmedos, y que prospera en condiciones agronómicas muy variadas, lo que permite su produción en tierras marginadas a otros cultivos, obteniéndose altos rendimientos. El grano de sorgo presenta composición química similar a la del maíz, por lo que la tecnología de procesamiento para la obtención de productos alimentarios e industriales a base de maíz o de otros cereales es aplicable al sorgo con la finalidad de explotar eficientemente su potencial como materia prima en la elaboración de diversos productos. La aplicación del proceso de extrusión, para la obtención de harinas pre-gelatinizadas y cereales instantáneos con diferentes propiedades de sabor, textura y formato, modificando sus propiedades funcionales; se considera como una tecnología de procesamiento adecuada para el grano de sorgo.
Los productos extruídos presentan larga vida de anaquel debido a las altas temperaturas usadas durante el proceso además de que ocurre una menor destrucción de nutrimentos y mejora la digestibilidad (Mans 1982, Rossem y Miller 1973).

El proceso de extrusión puede ser controlado mediante la manipulación de uno o más de las siguientes variables: temperatura en las diferentes zonas del extrusor, contenido de humedad de la materia prima, relación de compresión, tiempo de exposición, diámetro del dado y tamaño de partícula (Harper, 1989). Anderson et al. (1969), utilizaron el proceso de extrusión para gelatinizar harinas de sorgo. Los productos obtenidos presentaron mayores índices de absorción de agua a altos contenidos de humedad en la materia prima, y los índices de solubilidad en agua fueron mayores a bajos niveles de humedad. Señalando los autores, que productos con esas características son apropiados para preparación de bebidas y para usos industriales donde se requieran propiedades adhesivas.

\footnotetext{
* Trabajo presentado a la XXXVII Reunión del PCCMCA, Panamá. 1991.

**' Investigador del CIFAP-México, INIFAP. Apdo. Postal 10, Chapingo, Edo de México, cp. 56230.

***L íder del Programa de Sorgo ICRISAT-LASIP. Lisboa 27, Apdo. Postal 6-641 Colonia Juárez, Delegación Cuahutemoc, México. DF06600.
} 
Hay gran similitud en caracter ísticas funcionales presentadas por los productos (Harper, 1981) extruidos de sorgo obtenidos de "grits" con relación al maíz. Guerra (1985), indica la obtención por proceso de extrusión de productos expandidos de sorgo decorticado con baja densidad, indicando que las harinas pueden ser empleadas en la preparación de alimentos instantáneos o en diversos usos industriales (industria textil, papel y otras). Martínez (1988), utilizó el proceso de extrusión utilizando como materia prima sorgo integral y decorticado, para la obtención de harinas instantáneas para elaboración de tortillas.

\section{MATERIALES Y METODOS}

\section{Materia prima}

Sorgo blanco variedad Isiap Dorado, también liberado en México como variedad Blanco-86.

\section{Proceso de extrusión}

Fue utilizado un extrusor marca Mapinpianti con una capacidad de producción de $100 \mathrm{~kg} / \mathrm{h}$. Este equipo consta de 1 tornillo sin fin único conectado a un motor de $40 \mathrm{HP}$, con temperatura en la zona del dado de $110^{\circ} \mathrm{C}$, calentada mediante sistema de inducción eléctrica; dado de $2 \mathrm{~mm}$ de diámetro y velocidad de tornillo constante de $150 \mathrm{rpm}$.

El grano entero y libre de impurezas fue molido en un molino de cuchillas para obtener fracciones con diámetro de partícula de 350 micras, requerido para su procesamiento en este tipo de extrusor. Las harinas de sorgo decorticado fueron obtenidas sometiendo el sorgo integral al proceso de decorticado utilizando una pulidura de arroz (Satake) con un tiempo de residencia en la cámara de pulimiento de 1 minuto, y posteriormente molido el grano decorticado de acuerdo con el procedimiento seguido para la obtención de harina integral. Las harinas de sorgo integral y decorticado fueron acondicionadas a contenidos de humedad de 12 , 15 y $8 \%$, utilizando $5 \mathrm{~kg}$ de muestra en cada caso, se empleó una mezcladora (Hobart) a $1425 \mathrm{rpm}$ durante 10 minutos, y posteriormente las muestras fueron colocadas en bolsas de plástico con la finalidad de homogeneizar el contenido de humedad antes de someterlas al proceso de extrusión.

\section{Análisis físicos}

Se determinó el peso hectolítrico en $\mathrm{kg} / \mathrm{ha}$ y el peso de 1000 g. La dureza del grano se llevó a cabo en el durómetro Brabender. El color de grano y harinas fue determinado utilizando un colorímetro Hunter-Lab D25-2 (Hunter Associates Inc. Reston, VA). Las propiedades de viscosidad de las harinas se determinaron utilizando un viscoamilógrafo Brabender con $45 \mathrm{~g}$ de harina (14\% de humedad) en $450 \mathrm{ml}$ de agua. El índice de absorción de agua y el índice de solubilidad en agua de las harinas fueron llevados a cabo de acuerdo con la metodología descrita por Anderson et al. (1969). El grado de expansión de los productos extrudidos fue determinado midiéndose aleatoriamente el diámetro de 10 muestras y dividiéndose la media aritmética de los valores entre el diámetro del dado. La densidad de los extruidos se llevó a cabo pesando una parte lineal del producto de $5 \mathrm{~cm}$ de longitud dividiendo el resultado por su volumen.

\section{Análisis químicos}

Humedad método No. 45-5, cenizas método 08-03, nitrógeno método de Kjeldahl 46-10 usando el factor de 6.25 para la conversión de nitrógeno a proteína, AACC (1976). Fibra método 7,05 A (AOAC, 1975), grasa método de Bligh y Dyer (1959). Fenoles método de Burns (1971) y taninos método de Prince and Butler (1973).

\section{Análisis sensorial}

Las seis muestras de productos extruidos obtenidas de sorgo integral y decorticado procesadas con contenidos de humedad de 12, 15 y $18 \%$ fueron sometidas a una ordenación con 10 jueces no entrenados que ordenaron las muestras con respecto a una dada como referencia, basándose en parámetros de sabor, textura y apariencia general. Posteriormente, fueron seleccionados dos tratamientos obtenidos de la prueba de ordenación para realizar una prueba de aceptación por consumidores comparandolo con un producto comercial de caracter ísticas similares. Se utilizó una escala hedónica de 9 puntos donde 1 correspondió al concepto me disgusta extremadamente y 9 me gusta extremadamente, la prueba se realizó con 10 consumidores.

\section{RESULTADOS Y DISCUSIÓN}

\section{Características físicas de la materia prima}

En el Cuadro 1, se presentan los resultados obtenidos en peso hectolítrico, peso de 1000 granos, dureza y color en grano y harina de sorgo integral y decorticado. Los 
resultados obtenidos en peso hectolítrico y peso de 1000 granos se situan dentro de los valores ya obtenidos para sorgo, Guerra (1985) y Martínez (1988). Estas características son importantes en el transporte y almacenamiento del grano. Los valores obtenidos en el color de grano y harina lo sitúan como un grano blanco, ligeramente crema que mejoró su coloración blanca $(\mathrm{L}+)$ durante el proceso de decorticado.

\section{Composición química de la materia prima}

Los resultados sobre composición química de la materia prima obtenidos en grano integral y decorticado son presentados en el Cuadro 2. Estos resultados se situan dentro de los valores indicados para sorgo, Guerra (1985) y Martínez (1988), presentando el sorgo decorticado una reducción en el contenido de proteína, grasa, fibra, cenizas, taninos y fenoles.

\section{Composición química de los productos extruídos}

La composición química de los productos extruídos no fue alterada durante el proceso de extrusión, en general el color de los productos extruidos sufrió una reducción en el color blanco $(\mathrm{L}+)$, aumentando su color amarillo $(b+)$ en relación a sus respectivas harinas no tratadas, obteniéndose los valores más altos de $\mathrm{L}+$ (blanco) en productos obtenidos de sorgo decorticado y más amarillos (b+) en los obtenidos de sorgo integral (Cuadro 3).

\section{Propiedades de viscosidad}

Los índices de absorción de agua y solubilidad en agua de los productos extruidos (Cuadro 4), fueron modificadas durante el proceso de extrusión en relación a sus respectivas harinas no tratadas. No fue observado un comportamiento genérico en los productos extruidos con relación a sus propiedades de viscosidad, los índices de absorción de agua e índices de solubilidad en agua aumentaron posibilitando el empleo diversificado de estos productos. Estos resultados son similares a los informados en "grits" de sorgo (Guerra, 1985) y Harper,1981).

\section{Características de expansión y densidad de los productos extruidos}

Se considera que la expansión se produce cuando el valor del grado de expansión es por lo menos 1.5 (Harper,(1981). Es importante destacar, que la expansión de un producto es función compleja de varios factores, destacando la caracter ística elástica del producto, el tiempo que el producto permanece plástico en el extrusor, la intensidad y velocidad de evaporación del agua.

El análisis de varianza y comparación de medias (Cuadro 5), no mostró diferencias significativas $(\mathrm{P} \leq 0,05)$ entre tratamientos (12, 15 y $18 \%$ de humedad) entre sorgo integral y sorgo decorticado.Se obtuvieron productos menos densos en los provenientes de sorgo decorticado presentando diferencia significativa en relación a los productos obtenidos con sorgo integral, trigo integral y

Cuadro 1. Análisis físico y color de sorgo integral y decorticado.

\begin{tabular}{|c|c|c|c|c|c|c|c|c|c|c|}
\hline \multirow[b]{2}{*}{ Muestra } & \multirow{2}{*}{$\begin{array}{c}\mathrm{Ph} \\
(\mathrm{kg} / \mathrm{ha})\end{array}$} & \multicolumn{2}{|c|}{ Peso de } & \multirow{2}{*}{$\begin{array}{l}\text { Dureza } \\
\text { (seg.) }\end{array}$} & \multicolumn{3}{|c|}{ Color en grano 1/ } & \multicolumn{3}{|c|}{ Color en harina $1 /$} \\
\hline & & 100 & $\begin{array}{l}\text { granos } \\
(\mathrm{g})\end{array}$ & & L & a & $\mathrm{b}$ & L & a & $\mathrm{b}$ \\
\hline Sorgo Integral & 76,8 & & 27,55 & 19 & 56,8 & 2,8 & 17,1 & 74,9 & 0,1 & 11,2 \\
\hline Sorgo decorticado & 89,40 & & 20,50 & 16 & 69,4 & 0,0 & 19,6 & 81,0 & $-0,8$ & 9,4 \\
\hline
\end{tabular}

Cuadro 2. Composición química de sorgo integral y decorticado.

\begin{tabular}{lccccccc}
\hline Muestra & $\begin{array}{c}\text { Humedad } \\
(\%)\end{array}$ & $\begin{array}{c}\text { Proteina } \\
(\%)\end{array}$ & $\begin{array}{c}\text { Grasa } \\
(\%)\end{array}$ & $\begin{array}{c}\text { Fibra } \\
(\%)\end{array}$ & $\begin{array}{c}\text { Cenizas } \\
(\%)\end{array}$ & $\begin{array}{c}\text { Fenoles } \\
\text { mg ac. } \\
\text { tenico }\end{array}$ & $\begin{array}{c}\text { Equinos } \\
\text { catequina }\end{array}$ \\
\hline Sorgo Integral & 10,5 & 10,9 & 2,57 & 1,135 & 1,50 & 0,510 & 0,000 \\
Sorgo decorticado & 9,4 & 10,6 & 0,95 & 0,630 & 1,13 & 0,022 & 0,000 \\
\hline $\mathrm{n}=5$ & & & & & & & \\
\hline
\end{tabular}


"grits" de trigo las cuales presentaron menor grado de expansión, procesados en las mismas condiciones de operación, con menores valores de densidad en relación a sorgo,(Martínez y Salinas 1991). También los productos extruidos de sorgo integral y decorticado presentaron mayor grado de expansión en relación a los obtenidos por Guerra (1985), con sorgo decorticado, utilizando un extrusor de laboratorio Brabender, con diferentes condiciones de operación.

Cuadro 3. Composición química y color* de productos extruidos obtenidos del sorgo integral y decorticado.

\begin{tabular}{|c|c|c|c|c|c|c|c|c|}
\hline \multirow[b]{2}{*}{ Producto extruido } & \multirow{2}{*}{$\begin{array}{l}\text { Humedad } \\
(\%)\end{array}$} & \multirow{2}{*}{$\begin{array}{l}\text { Proteína } \\
\quad(\%)\end{array}$} & \multirow{2}{*}{$\begin{array}{c}\text { Grasa } \\
(\%)\end{array}$} & \multirow{2}{*}{$\begin{array}{c}\text { Fibra } \\
(\%)\end{array}$} & \multirow{2}{*}{$\begin{array}{c}\text { Cenizas } \\
(\%)\end{array}$} & \multicolumn{3}{|c|}{$\mathrm{C} \circ 1 \circ \mathrm{r}$} \\
\hline & & & & & & L & $\mathrm{a}$ & $\mathrm{b}$ \\
\hline $\begin{array}{l}\text { Integral extruido } \\
\text { con } 12 \% \text { de humedad }\end{array}$ & 9,1 & 10,9 & 2,50 & 1,130 & 1,50 & 70,6 & 0,6 & 15,0 \\
\hline $\begin{array}{l}\text { Integral extruido } \\
\text { con } 15 \% \text { de humedad }\end{array}$ & 9,7 & 10,9 & 2,50 & 1,130 & 1,50 & 69,6 & 0,4 & 15,2 \\
\hline $\begin{array}{l}\text { Integral extruido } \\
\text { con } 18 \% \text { de humedad }\end{array}$ & 10,5 & 10,9 & 2,50 & 1,130 & 1,50 & 69,3 & 0,4 & 14,0 \\
\hline $\begin{array}{l}\text { Decorticado extruido } \\
\text { con } 12 \% \text { de humedad }\end{array}$ & 9,1 & 10,6 & 0,93 & 0,625 & 1,13 & 76,1 & 0,5 & 14,7 \\
\hline $\begin{array}{l}\text { Decorticado extruido } \\
\text { con } 15 \% \text { de humedad }\end{array}$ & 9,5 & 10,6 & 0,93 & 0,625 & 1,13 & 72,8 & 0,6 & 14,5 \\
\hline $\begin{array}{l}\text { Decorticado extruido } \\
\text { con } 18 \% \text { de humedad }\end{array}$ & 9,7 & 10,6 & 0,93 & 0,625 & 1,13 & 78,3 & $-0,7$ & 12,4 \\
\hline
\end{tabular}

Cuadro 4. Propiedades de viscosidad, IAA, ISA de sorgo integral, decorticado y productos extruidos.

\begin{tabular}{|c|c|c|c|c|c|c|}
\hline \multirow[b]{2}{*}{ Muestra } & \multicolumn{4}{|c|}{$V i s c o s i d a d$} & \multirow[b]{2}{*}{ IAA } & \multirow[b]{2}{*}{ ISA } \\
\hline & $\overline{\mathrm{PT}}$ & $\mathrm{PV}$ & $\mathrm{V} 20 \mathrm{~min}$ & $\mathrm{~V} 50^{2} \mathrm{C}$ & & \\
\hline Sorgo Integral & 70 & 80 & 80 & 90 & 2,00 & 6,95 \\
\hline $\begin{array}{l}\text { Sorgo Integral extruido } \\
\text { con } 12 \% \text { de humedad }\end{array}$ & 0 & 0 & 0 & 0 & 7,75 & 28,20 \\
\hline $\begin{array}{l}\text { Sorgo Integral extruido } \\
\text { con } 15 \% \text { de humedad }\end{array}$ & 120 & 100 & 100 & 100 & 8,64 & 22,23 \\
\hline $\begin{array}{l}\text { Sorgo Integral extruido } \\
\text { con } 18 \% \text { de humedad }\end{array}$ & 60 & 60 & 60 & 60 & 7,83 & 18,00 \\
\hline Sorgo Decorticado $1 \mathrm{~min}$ & 73 & 60 & 140 & 200 & 1,59 & 6,48 \\
\hline $\begin{array}{l}\text { Sorgo Decorticado extruido } \\
\text { con } 12 \% \text { de humedad }\end{array}$ & 80 & 80 & 80 & 80 & 8,14 & 27,07 \\
\hline $\begin{array}{l}\text { Sorgo Decorticado extruido } \\
\text { con } 15 \% \text { de humedad }\end{array}$ & 90 & 90 & 90 & 90 & 8,40 & 28,15 \\
\hline $\begin{array}{l}\text { Sorgo Decorticado extruido } \\
\text { con } 18 \% \text { de humedad }\end{array}$ & 90 & 90 & 90 & 90 & 8,44 & 28,82 \\
\hline
\end{tabular}

\begin{tabular}{ll}
\hline $\mathrm{n}=3$ & PV : Pico de viscosidad (U.B.) \\
IAA : Indice de absorcion de agua & V20min: Viscosidad después de 20 min a $90^{\circ} \mathrm{C}$ \\
ISA : Indice de solubilidad en agua & V50 C : Viscosidad final a $500^{\circ}$ \\
PT: Temperatura de pasta ( $\mathrm{C})$ &
\end{tabular}

Cuadro 5. Comparación de medias del grado de expansión y densidad de los productos extruidos.

\begin{tabular}{|c|c|c|c|c|}
\hline & \multicolumn{2}{|c|}{ Expansión } & \multicolumn{2}{|c|}{ Densidad $(\mathrm{g} / \mathrm{cm} 3)$} \\
\hline & $\mathrm{n}$ & Media & $\mathrm{n}$ & Media \\
\hline Sorgo Integral & 30 & $3,238 a$ & 30 & $0,3624 a$ \\
\hline Sorgo decorticado & 30 & 3,205 a & 30 & $0,2477 \mathrm{~b}$ \\
\hline
\end{tabular}

Los valores de las medias en la misma columna con la misma letra no son significativamente diferentes $(\mathrm{P}<=0,05)$.
La comparación de medias por tratamientos (Cuadro 6 ), en el grado de expansión y densidad de los productos obtenidos presentó los mayores valores en expansión con los tratamientos de sorgo decorticado extrudido con $15 \%$ de humedad y sorgo integral con $18 \%$ de humedad; estos tratamientos también presentaron los más altos grados de expansión en el caso de trigos cristalinos integral y "grits", Martinez y Salinas (1991). Considerando estos resultados, estos productos fueron seleccionados para el análisis sensorial 
Cuadro 6. Comparación de medias por tratamiento del grado de expansión y densidad de los productos extruidos.

\begin{tabular}{|c|c|c|c|c|c|}
\hline & & \multicolumn{2}{|c|}{ Expansion } & \multicolumn{2}{|c|}{ Densidad $(\mathrm{g} / \mathrm{cm} 3)$} \\
\hline & & $\mathrm{n}$ & Media & $\mathrm{n}$ & Media \\
\hline \multicolumn{6}{|l|}{ Sorgo I } \\
\hline & $12 \%$ & 10 & $2,81 \mathrm{~b}$ & 10 & $0,3050 \mathrm{~b}$ \\
\hline & $15 \%$ & 10 & $3,20 \mathrm{~b}$ & 10 & 0,5612 \\
\hline & $18 \%$ & 10 & 3,70 a & 10 & 0,2210 \\
\hline \multicolumn{6}{|c|}{ Sorgo decorticado } \\
\hline & $12 \%$ & 10 & $3,28 \mathrm{~b}$ & 10 & 0,1140 \\
\hline & $15 \%$ & 10 & 3,76 a & 10 & 0,3755 \\
\hline & $18 \%$ & 10 & $2,46 \mathrm{c}$ & 10 & $0,2534 \mathrm{~b}$ \\
\hline
\end{tabular}

Los valores de las medias en la misma columna y con la misma letra no son significativamente diferente $(P<=0,05)$

\section{Prueba de aceptación}

En la prueba de aceptación de los tratamientos seleccionados, no existieron diferencias significativas entre los dos tratamientos. En general, los productos de sorgo mostraron buena aceptación, aunque menor que el producto comercial atribuído a que el producto comercial presentó una mayor concentración de saborizante que es preferido por el consumidor.

\section{CONCLUSIONES}

Los resultados obtenidos mostraron el potencial que representa el sorgo en la elaboración de productos expandidos o sus respectivas harinas, que pueden ser empleadas en diversos usos.

Durante el proceso de extrusión no se observaron cambios en su composición química, los productos obtenidos de sorgo decorticado presentaron mejores características tecnológicas con relación a los productos obtenidos de sorgo integral, aunque con el mismo grado de aceptación por los consumidores.

\section{LITERATURA CITADA}

AMERICAN ASSOCIATION OF CEREAL CHEMISTS (AACC). 1976. Aproved methods of the AACC. 9a. Ed. St. Paul Minn. USA.

ANDERSON, R. A; CONWAY, H. F.; PF'EIFF'ER, U.F.; GRIFFIN, RL 1969. Gelatinization of corn grits by rollo and extrusioncooking. Cereal Sci. Today. 14(1):4-7, 11-12.

ASSOCIATION OF OFFICIAL ANALYTICAL CHEMIST (AOAC). 1975. Official methods and purification. Cam. J. Byochen. 37:911-913.

BURNS, R. R 1971. Method of estimation of tannin in grain sorghum. Agron. J. 63:511-512.

BLIGH, R.E.; DYER, W. J. 1959. A rapid method of total lipid extration and purification. Can. J. Byochem. 37:911-913.

GUERRA M. M. J. 1985. Desenvolvimento de um processo de moagem de sorgo (Sorghum bicolor L. Moench) e de produccao de farinhas pre-gelatinizadas. Tese de Doutorado. Faculdade de Engenharia de Alimentos. UNICAMP. Brasil.

HARPER, J. M. 1981. Extrusion of foods. Vol I and II. CRC Press Inc. Boca Raton, Florida, USA.

HARPER, J. M. 1989. Food extruders and their applications, In: Extrusion cooking. Edited by Mercier, Ch., Linko, P., and Harper, 1.M. Published by the AACC, St. Paul, Minnesota, USA.

MANS, J. 1982. The la test breed can greatly increase line speed and consistency while paring production costs for a variety of prepared food. Prepared Food. 11:60-63.

MARTINEZ, B. F. 1988. Obtencao de farinhas instantaneas de sorgo para "tortillas" pelo processo de extrusao. Tese de Doutorado. Faculdade de Engenharia de Alimentos. UNICAMP. Brasil.

MARTINEZ, B. F.; SALINAS, M. Y. 1991. Extrusi6n de trigos cristalinos, III Congreso Agroindustrial. UACH. Chapingo, México.

PRINCE, M.L; BUTLER, L.G. 1973. Rapid visual estimations and espectrophotometric determination of tannin content of sorghum grain. J. Agri. Food Chem. 25(6): 1268-1273.

ROSSEM, J.L; MILLER, R.C. 1973. Food extrusion. Food Technology 27(8):46-53. 